\title{
ALS—dying forward, backward or outward?
}

\section{Mark R. Baker}

Heiko Braak and colleagues (Amyotrophic lateral sclerosis - a model of corticofugal axonal spread. Nat. Rev. Neurol. 9, 708-714; $2013)^{1}$ present impressively detailed evidence in support of corticofugal spreadalso known as the 'dying-forward' model of neurodegeneration, first proposed by Eisen and Weber ${ }^{2}$-in amyotrophic lateral sclerosis (ALS). Here, I propose a refinement that integrates both the 'dying-forward' and 'dying-back' ${ }^{3}$ models: the corticofugal synaptopathy, or 'dying-outward' hypothesis.

In any model of ALS, a number of fundamental features have to be reconciled. First, degenerative changes occur primarily in anterior horn cells and brainstem motor neurons that receive monosynaptic connections from the motor cortex, ${ }^{4}$ and in the corticospinal tract neurons within the primary motor cortex. Second, in some variants of ALS, the disease only affects the corticospinal tract neurons, ${ }^{5}$ whereas in other variants, it only affects anterior horn cells, or affects corticospinal tract neurons only very late in the disease. ${ }^{6}$ Third, ALS progresses contiguously between spinal, brainstem and cortical regions, in what has been termed a 'prion-like' pattern. ${ }^{7}$ Fourth, cortical areas involved late in the disease are linked via long-range synaptic connections. ${ }^{1}$ Last, humans are the only species affected by sporadic ALS, and only nonhuman primate models of ALS have recapitulated features of the disease observed in humans. ${ }^{8}$

An important component of the corticofugal model ${ }^{1}$ is the axonal transport hypothesis, which identifies the importance of long-range axonal connections in disease propagation, but overlooks the synapsethe very reason for the existence of such connections. Not only does the developing synapse, or growth cone, function independently, ${ }^{9}$ but there is also evidence that synaptic autonomy continues into adulthood. ${ }^{10}$ For example, synaptic prion-like proteins maintain activity-dependent changes in synaptic efficacy independently of nuclear transcription within neuronal somata. Furthermore, mitochondria, which are essential for calcium buffering and energy production, are maintained autonomously within the presynaptic and postsynaptic compartments. ${ }^{11}$ Such autonomy permits efficient long-distance neuronal communication, but there is a trade-off: the lysosomal housekeeping processes that are responsible for recycling biomolecules, organelles and cellular debris located within the distant soma function less efficiently. Consequently, abnormal conformational changes in prionlike proteins can replicate and propagate without control, and dysfunctional mitochondria accumulate. ${ }^{10,11}$ The longer the axon and the larger the synapse, the more likely this autonomous process is to malfunction, hence the susceptibility of the monosynaptic corticomotoneuronal synapse at the onset of ALS in humans.

The corticomotoneuronal synapse is a feature that distinguishes primates from other mammalian species, and the number of corticomotoneuronal synapses and length of axons in the corticospinal tract distinguish humans from nonhuman primates. ${ }^{12}$ Mutations in mitochondrial DNA have been implicated in motor neuron diseases ${ }^{13}$ and ALS, ${ }^{14}$ and there is increasing evidence that the interaction between pathological synaptic mitochondria and synaptic prion proteins leads to neurodegeneration. ${ }^{11}$ The corticomotoneuronal synapse, therefore, is not only pivotal as the link between the corticospinal tract and anterior horn cells; because of its vulnerability, it is also an efficient nidus for neurodegeneration. Consequently, biomarkers that can detect changes in the integrity of the corticomotoneuronal synapse ${ }^{15}$ should be able to identify the very earliest stages of ALS, enabling early disease-modifying therapeutic interventions at a stage when they can make a significant impact on survival in this dreadful disease.

Institute of Neuroscience, Henry Wellcome Building, The Medical School, Newcastle University, Framlington Place, Newcastle upon Tyne, NE2 4HH, UK.

Correspondence to: M. Baker

mark.baker@newcastle.ac.uk
Competing interests

The author declares no competing interests.

1. Braak, H. et al. Amyotrophic lateral sclerosis-a model of corticofugal axonal spread. Nat. Rev. Neurol. 9, 708-714 (2013).

2. Chou, S. M. \& Norris, F. H. Amyotrophic lateral sclerosis: lower motor neuron disease spreading to upper motor neurons. Muscle Nerve 16, 864-869 (1993).

3. Hudson, A. J. \& Kiernan, J. N. Preservation of certain voluntary muscles in motoneurone disease. Lancet 1, 652-653 (1988).

4. Pringle, C. E. et al. Primary lateral sclerosis. Clinical features, neuropathology and diagnostic criteria. Brain 115 495-520 (1992).

5. Ince, P. G. et al. Corticospinal tract degeneration in the progressive muscular atrophy variant of ALS. Neurology 60 , 1252-1258 (2003).

6. Kanouchi, T., Ohkubo, T. \& Yokota, T. Can regional spreading of amyotrophic lateral sclerosis motor symptoms be explained by prion-like propagation? J. Neurol. Neurosurg. Psychiatry 83, 739-745 (2012).

7. Eisen, A. \& Weber, M. The motor cortex and amyotrophic lateral sclerosis. Muscle Nerve 24, 564-573 (2001).

8. Uchida, A. et al. Non-human primate model of amyotrophic lateral sclerosis with cytoplasmic mislocalization of TDP-43. Brain 135, 833-846 (2012).

9. Kater, S. B. \& Rehder, V. The sensory-motor role of growth cone filopodia. Curr. Opin. Neurobiol. 5, 68-74 (1995).

10. Raveendra, B. L. et al. Characterization of prion-like conformational changes of the neuronal isoform of Aplysia CPEB. Nat. Struct. Mol. Biol. 20, 495-501 (2013).

11. Siskova, Z. et al. Morphological and functional abnormalities in mitochondria associated with synaptic degeneration in prion disease. Am. J. Pathol. 177, 1411-1421 (2010).

12. Lemon, R. N. Descending pathways in motor control. Annu. Rev. Neurosci. 31, 195-218 (2008).

13. Dion, P. A., Daoud, H. \& Rouleau, G. A. Genetics of motor neuron disorders: new insights into pathogenic mechanisms. Nat. Rev. Genet. 10, 769-82 (2009).

14. Borthwick, G. M. et al. Motor neuron disease in a patient with a mitochondrial tRNAlle mutation. Ann. Neurol. 59, 570-574 (2006).

15. Fisher, K. M., Zaaimi, B., Williams, T. L., Baker, S. N. \& Baker, M. R. Beta-band intermuscular coherence: a novel biomarker of upper motor neuron dysfunction in motor neuron disease. Brain 135, 2849-2864 (2012). 